\title{
DEVELOPMENT OF FUZZY LOGIC BASED MODE CHOICE MODEL CONSIDERING VARIOUS PUBLIC TRANSPORT POLICY OPTIONS
}

\author{
Mukesh Kumar', Pradip Sarkar ${ }^{2}$, Errampalli Madhu ${ }^{3}$ \\ ${ }^{1}$ Department of Transport and Infrastructure, WSP Group, New Delhi, India \\ ${ }^{2}$ Department of Transport Planning, School of Planning and Architecture, New Delhi, India \\ ${ }^{3}$ Transport Planning and Environment Division, Central Road Research Institute, New Delhi, India
}

Received 5 March 2013; accepted 16 September 2013

\begin{abstract}
Mode choice modeling has been studied by transport planners in many researches' applications. This study explores the relationships between travel patterns of commuters and their willingness to adopt an alternate mode. The study also estimated public transport share along with private vehicles. This research has been concentrated on the application of fuzzy logic technique in which the mode choice model provides the estimated modal split at the specified work centers in the study area. It is expected that the present study may be used in new transport policies by concerned authorities.
\end{abstract}

Keywords: mode choice model, fuzzy logic, public transport policy.

\section{Introduction}

There are a number of studies carried out by various researchers in the past on mode choice model. Most of the models reported so far are based on behavioral model focusing mainly on the development of Logit model. Further, this has been extended to either using Probit model or discriminant analysis. The selection of mode choice is not always governed by the objective function. It has been observed that the fuzziness prevails in the mind of choice riders in selecting the appropriate mode of transport. The degree of uncertainty is very difficult to be quantified. Keeping this in view, in this paper, an attempt has been made to develop a mode choice model based on fuzzy logic technique. In order to develop a fuzzy logic model, three work centers namely Netaji Subhash Place, Nehru Place and Connaught Place in Delhi (India) urban area was considered.
The primary purpose of the paper is to test number of public transport policy variables to estimate the modal share with respect to personalized and public transport modes.

The paper is organized as follows: Section 1 introduced with technique used for carrying out the study in the given study area; Section 2 provides detailed introduction to study area and its characteristics; In Section 3, methodology of the study has been explained through schematic flow diagram; Section 4 presents a relevant literature review on different studies carried out using fuzzy technique; Section 5 explains the concept of fuzzy logic technique; Section 6 provides the analysis of commuter interview survey; Section 7 describes the application of fuzzy logic technique in mode choice model; Section 8 provides the ideal shape of membership function levels for both input and output; In Section 9, execution

${ }^{1}$ Corresponding author: mukesh.dhawan@wspgroup.in 
of fuzzy inference system to evaluate output by product-sum gravity approach has been described; Section 10 presents the prediction accuracy for both the calibrated and validated fuzzy logic based mode choice models; In Section 11, estimated modal-split of study area (Delhi) by FloMoChoMo has been presented; Section 12 describes the policy sensitivity analysis using different policy options; Section 13 gives the various conclusions of the study.

\section{Study Area Characteristic}

Delhi, the National Capital Territory of Delhi (NCT), is the largest metropolis by area and the second-largest metropolis by population in India. It is the eighth largest metropolis in the world by population with 16.7 million inhabitants in the territory at the 2011 Census. The Delhi Metro, a mass rapid transit system built and operated by Delhi Metro Rail Corporation (DMRC), serves many parts of Delhi as well as the satellite cities area like Gurgaon in the neighboring Haryana, Noida, and Ghaziabad in neighboring Uttar Pradesh. Personalized vehicles account for $30 \%$ of the total demand for transport.

Delhi has one of the highest road densities in India. Buses are the most popular means of transport catering to about $40 \%$ of the total demand. The state-owned Delhi Transport Corporation (DTC) is a major bus service provider for the city. The DTC operates the world's largest fleet of environment friendly CNG buses. BRTS in Delhi runs between Ambedkar Nagar and Delhi Gate. Three survey locations were identified in study area Delhi to conduct commuter interview survey. All three work centers are very well connected by means of public transport via Delhi Metro \& DTC bus service. Three profile of work centers are further described in details.

\subsection{Netaji Subhash Place District Centre}

Netaji Subhash Place is a new financial, commercial and business center in Delhi which is growing rapidly. The nearest Metro Station includes Netaji Subhash Place station on Red line. The place is very well connected to Delhi Metro \& DTC Bus service and is located between Inner and Outer ring road, $\mathrm{GT}$ road and Rohtak road.

\subsection{Nehru Place District Center}

Nehru Place District Center is also one of the largest financial, commercial and business centers in Delhi. The nearest Metro Stations includes Nehru Place and Kalkaji Mandir on Violet Line (Central Secretariat to Badarpur). It has its own bus terminal, popularly known as Nehru Place District Center bus terminal.

\subsection{Connaught Place}

Connaught Place is one of the largest financial, commercial and business centers in Delhi. It is connected by Rajeev Chowk Metro Station to other parts of Delhi and is very well connected by DTC bus service.

\section{Methodology of the Study}

The literature review of fuzzy logic technique has been made so that the concept of the technique can be appreciated and applied. After formulation of the objectives and selection of the survey locations in study area, the survey format was designed that includes all necessary information about commuters and their trip details to develop mode choice model. The collected data 
was used for comparative analysis among three survey locations on the basis of socioeconomic and trip characteristics.

At later stage, the different data sets were prepared to develop mode choice models. The developed fuzzy logic based mode choice model was further applied for policy sensitivity analysis by introducing new transport policies namely Policy 1, 2, 3, 4, 5 and 6 . Following this, comparison of the estimated modal split among all policies in favor of improvement in public transport share has been made. This is carried out to evaluate the estimated modal split of study area using fuzzy logic mode choice model. Fig. 1 shows the various activities of the study in form of flowchart.

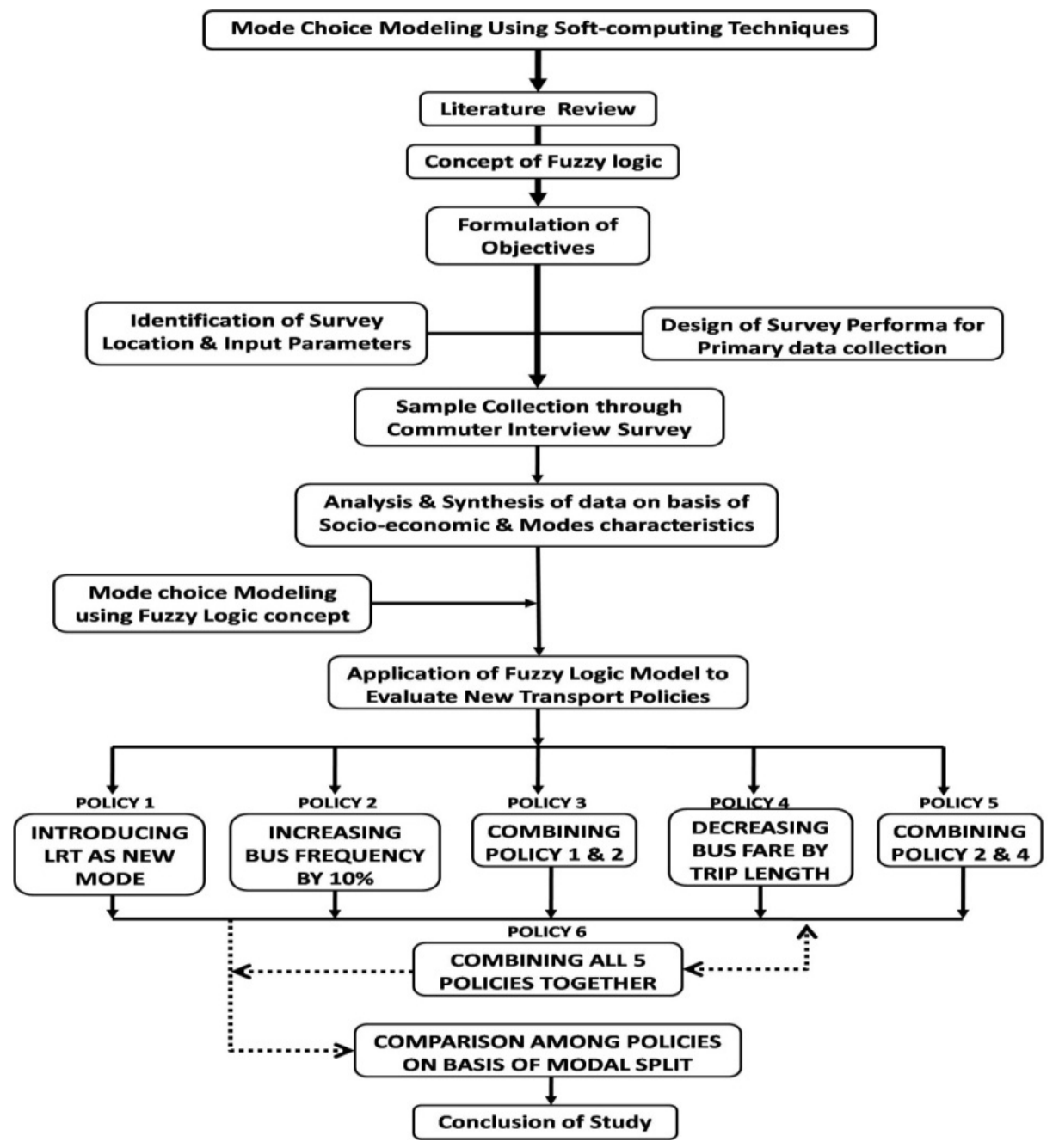

Fig. 1.

Methodology of the Study 


\section{Literature Review}

From the past years, mode choice criteria have been major vital research area for researcher due to its effectiveness through different parameters at various cities. Therefore, a number of research works have been carried out by researchers for issues related to mode choice modeling by using fuzzy logic technique. Few studies have been discussed in this section.

Madhu (2008) carried out a study were various models were developed, e.g. vehicle generation model, lane assign model, parking area movement model, intersection movement model, bus stop/ LRT stop behavior model. Madhu (2008) also used fuzzy logic technique to develop fuzzy logic based car-following model and route choice model. This study also includes microscopic traffic simulation to describe transport policy evaluation.

Postorino and Versaci (2008) worked on fuzzy approach to simulate the user mode choice behavior. In this study, they used fuzzy approach starting from the hypothesis that the complex human decision mechanism can be well represented by fuzzy sets which simulate the user mode choice behavior. The good results obtained in this study confirm the initial hypothesis and suggest more research work in this field using fuzzy approach.

Nath (2000) developed a mode choice model using fuzzy set theory for study area Delhi in India. This research work has been designed to study the travel mode choice behavior modeling by a non-conventional approach using fuzzy measures. Stated preference data set has been used to identify various income groups of commuters in terms of their selection of predominant modes.
Dhingra and Ramanajee (1998) presented a research paper on evaluation of utility of mode using fuzzy integrals. This study is limited to the mass transit modes only. Dhingra and Ramanajee (1998) developed a model using fuzzy integrals based on stated preference data sets collected via commuter interview survey. This study explained an extensive use of fuzzy integrals in mode choice modeling through function of utility of mode of travel among various mass transit modes. The function of utility has been evaluated by parameters influencing choice of a mode of travel.

\section{Concept of Fuzzy Logic Technique}

\subsection{Introduction}

The fuzzy logic technique was introduced in 1965 by Lotfi Zadeh. The fuzzy logic provides a mechanism for representing linguistic variables such as "many", "low", "medium", "often", "few", etc. On the contrary, the traditional binary set theory describes crisp events, events that either do or do not occur (Madhu, 2008). The theory of fuzzy logic is based upon the concept of relative graded membership. It is important to observe that there is an intimate connection between Fuzziness and Complexity. As the complexity of a task (problem), or of a system for performing that task, exceeds a certain threshold, the system must necessarily become fuzzy in nature. Real world problems (situations) are too complex, and the complexity involves the degree of uncertainty - as uncertainty increases, so does the complexity of the problem. In respect to this, fuzzy systems theory is similar to other engineering theories, because almost all of them characterize the real world in an approximate manner. Fuzzy logic provides means to model the 
uncertainty associated with vagueness, imprecision, and lack of information. Consider the meaning of a "short person", for an individual $\mathrm{X}$, the short person may be one whose height is below 4'25”. For other individual $\mathrm{Y}$, the short person may be one whose height is below or equal to 3'90'. This "short" is called as a linguistic descriptor. The term "short" is the same meanings to the individuals $\mathrm{X}$ and $\mathrm{Y}$, but it is found that they both do not provide a unique definition. The term "short" would be conveyed effectively, only when a computer compares the given height value with the pre-assigned value of "short." This variable "short" is called as linguistic variable, which represents the fuzziness existing in the system. Lotfi Zadeh proposed the set membership idea to make suitable decisions when uncertainty occurs. Consider the "short" example discussed previously. If we take "short" as a height equal to or less than 4 feet, then 3'90" would easily become the member of the set "short" and 4'25" will not be a member of the set "short". The membership value is " 1 " if it belongs to the set or " 0 " if it is not a member of the set. Thus membership in a set is found to be binary i.e., the element is a member of a set or not. Thus membership in a set is found to be binary i.e., the element is a member of a set or not. It can be indicated as:

$\boldsymbol{\mu}_{A(x)}=\left(\begin{array}{ll}1 & x \in A \\ 0 & x \notin A\end{array}\right)$

Where $\mu_{A}(x)$ is the membership of element $x$ in set $A$ and $A$ is the entire set on the universe. This membership was extended to possess various "degree of membership" on the real continuous interval $[0,1]$. Zadeh generalized the idea of a crisp set by extending a valuation set $\{1,0\}$ to the interval of real values (degrees of membership) between 1 and 0 denoted as [0,1]. He says that the degree of membership of any particular element of a fuzzy set express the degree of compatibility of the element with a concept represented by fuzzy set. It means that a fuzzy set $A$ contains an object $x$ to degree $a(x)$, i.e., $a(x)=\operatorname{Degree}(x \in A)$ and the map $a: X \rightarrow\{$ Membership Degrees $\}$ is called a set function or membership function. A fuzzy set $A$ on universe $X$ is a set defined by a membership function $\mu_{A}$ that can be defined by a mapping:

$$
\boldsymbol{\mu}_{A ;(X) \rightarrow(1,0)}
$$

Here the value of $\mu_{A}(x)$ for the fuzzy set $A$ is called the membership value or the grade of membership of $x \in X$. The membership value represents the degree of belongingness to the fuzzy set $A$.

\subsection{Membership Functions}

In fuzzy logic technique, linguistic terms are used to evaluate the output in which posting of membership function to the variables is carried out. For example, in a crisp set of travel time "medium" denotes that travel to destinations is $30 \mathrm{~min}$ or less but in case of fuzzy set it indicates that travel time to destination is "medium" that is about $30 \mathrm{~min}$. This means that it may be less than $30 \mathrm{~min}$ or may be more than $30 \mathrm{~min}$. An example of membership function for travel time has been explained in Fig. 2. 

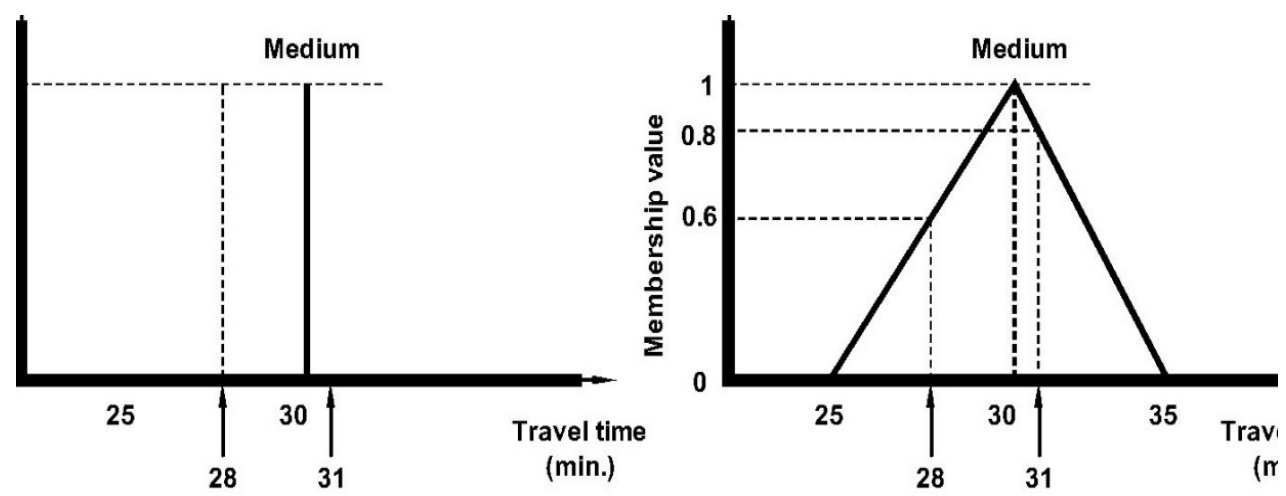

Fig. 2.

Description of Crisp Set and Fuzzy Set for Travel Time "Medium"

Assume that travel time value is $28 \mathrm{~min}$ to destination. So as per crisp set, it is considered as medium with membership value 1 because it is falling left side of $30 \mathrm{~min}$ vertical line as shown in crisp set graph in Fig. 2. If the travel time is $31 \mathrm{~min}$, then the membership value is zero as per the crisp set because it is falling right side of the $30 \mathrm{~min}$ vertical line and $31 \mathrm{~min}$ is no longer called as medium travel time. In case of fuzzy set, a triangular shape of membership function for "medium" has been shown in fuzzy set graph in Fig. 1. Here, the travel time of 28 min and $31 \mathrm{~min}$ is spread along both sides of $30 \mathrm{~min}$ with membership value 0.6 and 0.8 respectively as shown in Fig. 2 . The middle value (controlled value) of $30 \mathrm{~min}$ decides whether the time is medium or not for crisp set. In case $31 \mathrm{~min}$ which is only one minute more than $30 \mathrm{~min}$ value, the crisp set rejects that value as "medium" travel time, while fuzzy set can accept this value as "medium" with certain specified weight or membership value. In case of crisp set, output can be affected by small differences. In real situations a user can not estimate the exact value of travel time but can estimate the travel time on the basis of some linguistics terms such as "large", "medium" or "small" and also the users make some approximations for value of travel time which may have some variations as compared to exact value of travel time. Hence, if crisp set is used for such approximated data, then, it can lead to incorrect estimation of conclusions from the situations where human behavior is concerned. In the same case, fuzzy logic responds rationally with such kind of situations and is very useful than crisp set. Due to this reason; fuzzy logic has the ability to tackle such kind of small variations and considered approximations (Madhu, 2008). Therefore, fuzzy logic is very helpful in the situations where exact information is not available or difficult to get. In case of mode choice for work trip, it is difficult to choose a particular mode when two modes with more or less similar characteristics are compared with each other. Hence, application of fuzzy logic technique to develop the mode choice model based on commuter's choice criteria can efficiently give better results.

\subsection{Fuzzy If-Then Rule Bases}

The fuzzy logic systems consist of if-then rules that specify a relationship between the 
input and output variables. The fuzzy rule is generally in the following form:

IF Travel cost in mode ' $\mathrm{X}$ ' is Very Big AND Comfort level in mode ' $\mathrm{X}$ ' is Very Low

THEN Utility of mode ' $\mathrm{X}$ ' is Very small.

A fuzzy rule base contains sets of fuzzy ifthen rules. A single if-then rule assumes the form "If $x$ is $T_{x}$ then $y$ is $T_{y}$ ". Examples of if-then rules used in the study are as follows:

Rule 1: "IF Travel cost is Very Big and Comfort level is Very High THEN Utility is Medium", from this it can be said that the mode choosing probability would also become "medium".

Rule 2: "IF Travel cost is Very Big and Comfort level is High THEN Utility is Medium", from this it can be said that the mode choosing probability would also become "medium".

Rule 3: "IF Travel cost is Very Small and Comfort level is Very High THEN Utility is Very Big", from this it can be said that the mode choosing probability would also become "very big".

Rule 4: "IF Travel cost is Small and Comfort level is Very Low THEN Utility is Medium", from this it can be said that the mode choosing probability would also become "medium".

From above statements, it can be observed that the trip maker first thinks on travel cost and then on comfort to estimate utility of that particular mode.

\subsection{Fuzzy Inference System (FIS)}

In this system, input is given as crisp values and is processed for fuzzification as per membership function. In fuzzyfication process, every input is being analyzed according to different membership functions. After fuzzification they are further analyzed on the basis of fuzzy ifthen rules in inference engine in which product-sum gravity techniques is used to generate output, but the generated output is in the linguistic form which cannot be used directly. To make them usable, a process of defuzification is used to convert output into crisp value (Madhu, 2008). Fig. 3 shows the schematic diagram of fuzzy inference system.

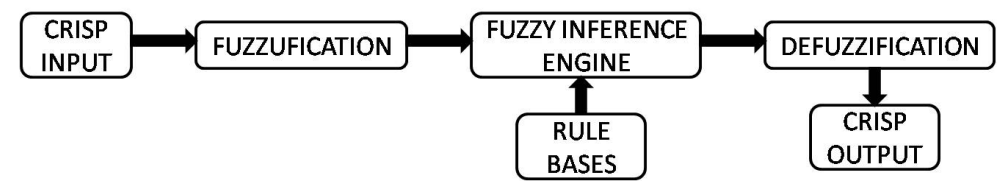

Fig. 3.

Schematic Diagram of Fuzzy Inference System

\subsection{Defuzzyfication}

Defuzzyfication means the fuzzy to crisp conversions. The output generated by fuzzy logic technique cannot be used as such to the applications; hence it is necessary to convert the fuzzy quantities into crisp quantities for further processing. This can be achieved by using defuzzyfication process. The input to the defuzzyfication process is a fuzzy set (the aggregated output fuzzy set), and the output of the defuzzyfication process is a single number. Many defuzzyfication techniques have been proposed by researchers. The most commonly used method is the centroid. Other methods include maximum 
height, means of maxima, and modified height method. The output of an entire fuzzy process can be union of two or more fuzzy membership functions (Hellmann, 2000). The Centroid method analyzes the combined shape of membership function which gives us comparatively good results as compared to other methods. In this method, if there are numbers of parameters given and each parameter have its own specified membership function then, final shape of membership function is analyzed as per specified fuzzy rule to give the result of membership value on the basis of center of gravity of shape of membership functions developed from different membership function parameters (Akiyama and Okushima, 2010).

\section{Data Analysis}

Data analysis is carried out in order to assess socio-economic and trip characteristics of commuters in study area. The motive of analysis is to assess distinctive travel behavior of commuters at three work centers in study area. At Netaji Subhash Place, majority of $52.79 \%$ commuters belongs to Rs.20,000-30,000 income group while at Nehru Place, $42 \%$ commuters belongs to Rs.20,000-30,000 income group and at Connaught Place, $29.50 \%$ commuters belong to Rs.20,000-30,000 income group. It is seen that car owner group constitutes a share $5 \%$, $4 \%$ and $19 \%$ at Netaji Subhash Place, Nehru Place and Connaught Place respectively. This is mainly due to less share of commuters belonging to high income group as discussed earlier, while 2 -wheeler owner group constitutes a highest share of $57 \%, 59 \%$ and $37 \%$, respectively at above three places. This is due to fact that most of commuters belong to middle income group. Here, modal split at Netaji Subhash Place indicates that personalized vehicles accounts for a share of $55 \%$ with public transport constituting of $41 \%$ share, at Nehru place along with personalized vehicles having share of $40 \%$ while public transport constitutes $57 \%$ share (in view of public transport, buses including charted bus service exhibits a large share of $40 \%$ which is much larger than $17 \%$ share of metro service). Therefore, less percentage of commuters uses metro service while high percentage of commuters uses bus services at Nehru Place. Modal split at Connaught Place indicates that personalized vehicles accounts for a share of $41 \%$ while public transport accounts for $57 \%$ share. In view of public transport, metro service constitutes a large share of $39 \%$ which is much larger than $18 \%$ share of bus service. This is less as compared to personalized vehicle share. It implies that there is a need to improve public transport services at Netaji Subhash Place. Inferences can be drawn from analysis of data on the basis of average in-vehicle travel time, average out-of-vehicle travel time and average travel cost by each mode. This would help to make a definite comparison among public transport services. Netaji Subhash Place experiences highest out-of vehicle travel time of $28 \mathrm{~min}$ and $27 \mathrm{~min}$ for both bus and metro service respectively as it has comparatively lesser public transport service. The analysis of locations specific data with respect to in-vehicle travel time among personalized vehicles and public transport services implies that both Nehru Place and Connaught Place record comparatively higher in-vehicle travel time for bus. In case of in-vehicle travel time for personalized vehicles at Nehru Place, records highest in-vehicle travel time as 40 min. Nehru Place District Center has largest average trip length of $14.7 \mathrm{~km}$ as compared to other two locations. Connaught Place has second largest average trip length of $14.5 \mathrm{~km}$. In case of metro service at Netaji Subhash 
Place and Nehru Place, they experience quite similar results of $36 \mathrm{~min}$ and $38 \mathrm{~min}$, respectively which are larger as compared to 26 min with respect to Nehru Place. In context of travel cost, Netaji Subhash Place records highest average travel cost of Rs.33 incurred by personalized vehicles users.

\section{Application of Fuzzy Logic in Mode Choice Model}

After understanding concept of fuzzy logic technique, this technique is applied

\section{Table 1}

Influencing Input Variables

\begin{tabular}{|l|l|}
\hline \multicolumn{2}{|l|}{ Input parameters } \\
\hline OVTT & Out of Vehicle Travel Time \\
\hline IVTT & In Vehicle Travel Time \\
\hline TC & Travel Cost \\
\hline CFT & Comfort Level \\
\hline INC & Income Level \\
\hline
\end{tabular}

in development of mode choice model. Mode choice model is based on commuter's vehicle ownership criteria. To develop mode choice model, total five input variables have been considered which affect mode choice behavior of commuters. Table 1 and Table 2 present input variables and their membership function level.

In this case study, two input variables are considered in each rule for output as utility (UTL). Every input variable has five membership levels as shown in Table 2 .

\section{Table 2}

Membership Function Levels of Variables

\begin{tabular}{|l|l|l|l|l|l|}
\hline $\begin{array}{l}\text { Level of membership function } \\
\text { for INC, TC, Utility }\end{array}$ & \multicolumn{2}{l|}{$\begin{array}{l}\text { Level of membership function } \\
\text { variables for OVTT, IVTT }\end{array}$} & \multicolumn{2}{l|}{$\begin{array}{l}\text { Level of membership function } \\
\text { variables for CFT }\end{array}$} \\
\hline VSL & Very small & VLW & Very low & VLS & Very less \\
\hline SL & Small & LW & Low & LS & Less \\
\hline MED & Medium & AVG & Average & MED & Medium \\
\hline BG & Big & HG & High & LG & Large \\
\hline VBG & Very big & VHG & Very high & VLG & Very large \\
\hline
\end{tabular}

There are total 6 combination of rules that have been considered which are "TC with CFT”, “TC with OVTT”, “TC with IVTT”, "INC with OVTT", "INC with TC" and "INC with CFT". Hence, total 150 if-then rules are developed for execution of FIS. The process for application of fuzzy logic in mode choice model has been shown in Fig. 4. After preliminary stage, the input files has been prepared for development of mode choice model through program based on visual basic language Program uses input files to calibrate and validate model. Program generates output with respect to prediction accuracy of model and estimated model split of study area. In this study, a total 20 sets of 150 different if-then rule bases have been made. It means that a total 3000 if-then rules are used to develop mode choice model using fuzzy logic technique. Fig. 4 shows formulation of fuzzy logic based mode choice model using input variables with their membership functions in FIS. 


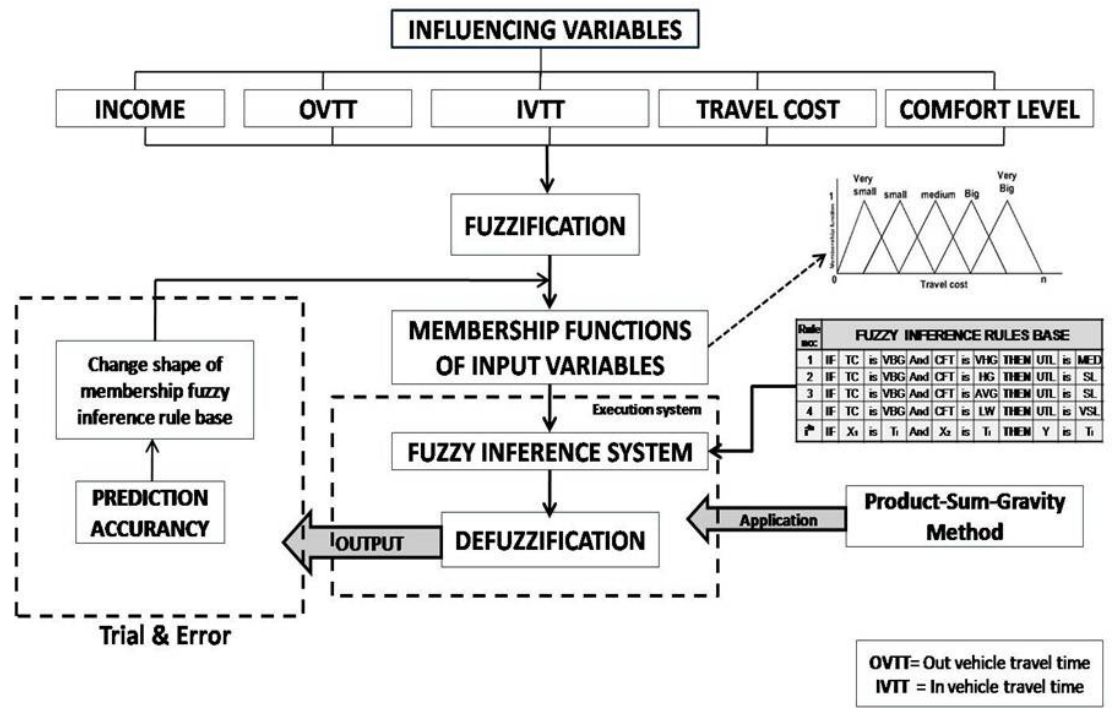

Fig. 4.

Flowchart of Calibration Process in Fuzzy Logic Technique

\section{Shape of Membership Function Levels for both Input and Output}

Membership functions are based on perception for labeling the input variable so that functioning of input variable can be known by its membership function shape. In this study, a triangular shape of membership function is considered for both input and output parameters as shown in Fig. 5.
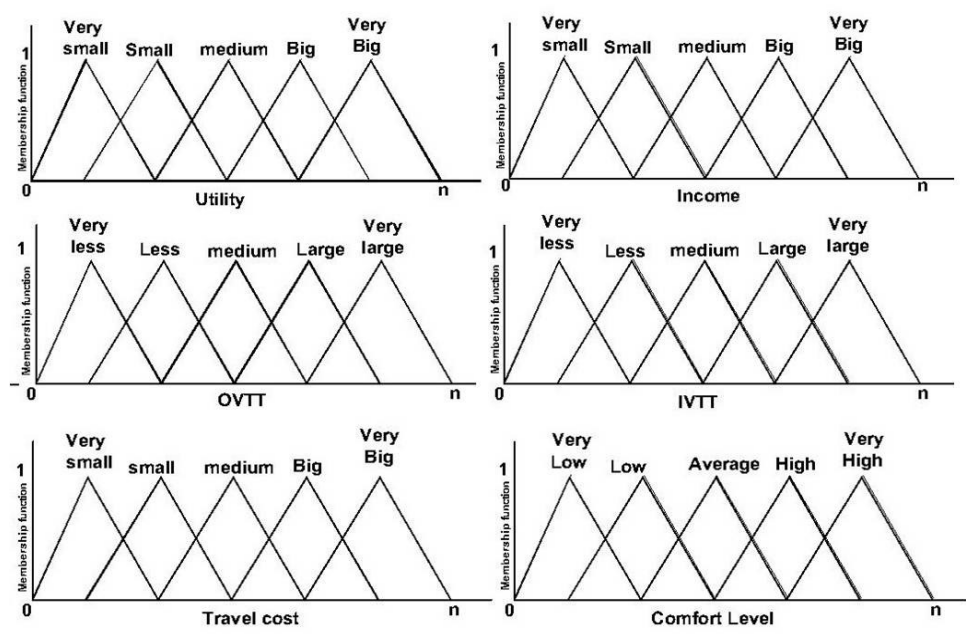

Fig. 5.

Shapes of Levels of Membership Function of all Parameters 


\section{Model Development}

\subsection{Execution of Fuzzy Inference System to Evaluate Output by Product-Sum Gravity Approach}

An example of choosing a mode can be shown by considering Fig. 6, two rules have been shown to elaborate the process of productsum-gravity approach (Madhu, 2008).

Rule 1: "IF Travel cost (X1) is Big (A1) and Comfort (Y2) is Medium (B1) THEN Utility (Z1) is Medium (C1)", from this it can be said that the mode choosing probability would also become low. As can be seen in the rule 1, the membership values for $X 1$ with $Y 2$ are 0.7 and 0.4 respectively. In product sum gravity approach, product of both the input variables is taken to evaluate the output membership value. Therefore, rule 1 presents the output membership value, i.e. $\mathrm{Cl}$ as 0.28 .
Rule 2: "IF Travel cost (X2) is Medium (A2) and Comfort (Y2) is High (B2) THEN Utility (Z2) is Medium (C2)", from this it can be said that the mode choosing probability would also become medium. Rule 2 presents output membership value, i.e. $C 2$ as 0.4 . Now, to evaluate final output $(Z)$ as membership value, take the sum of $C 1$ and $C 2$ which gives the final output membership value, i.e. $C 3$ as 0.68 . The advantages of product-sum-gravity approach areas under:

1. This approach uses membership value of both input variables by multiplying both the values together for evaluation of output membership value.

2. Both lesser and higher membership values are used of each input variables due to which each rule is used for development of final output membership value.

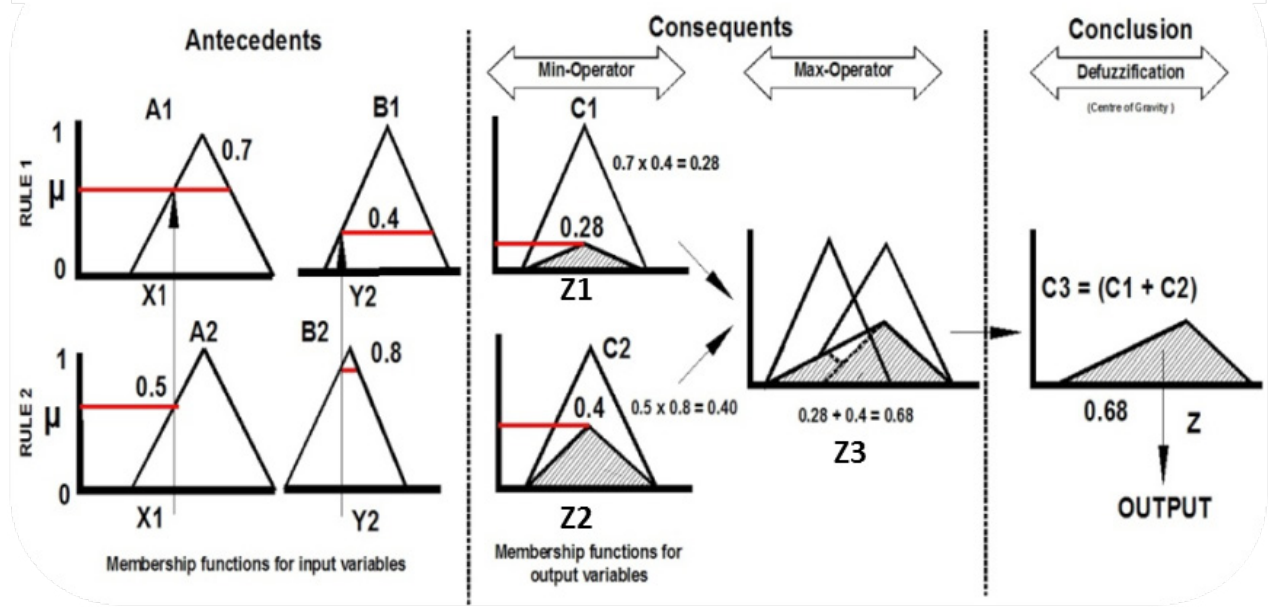

Fig. 6.

Product-Sum-Gravity Approach 


\section{Prediction Accuracy of Fuzzy Logic Based Mode Choice Model}

The fuzzy logic based mode choice model is described as "FloMoChoMo" in this case study. At this stage, overall prediction accuracy of fuzzy logic final model is presented in Table 3. From Table 3 it can be seen that 50, $116,7,95,126$ and 0 samples are observed for selection of car, 2-wheeler, cycle, bus, metro and auto-rickshaw, respectively. But fuzzy logic mode choice model estimated a total sample of $72,165,9,43,106$ and 1 , with respect to car, 2-wheeler, cycle, bus, metro and autorickshaw, respectively. Prediction accuracy of the model was estimated using number of samples correctly classified considering both observed and estimated choices. Analysis carried out demonstrates an accuracy of about $62 \%$ of calibrated samples as shown in Table 3 .

Table 3

Calibrated Prediction Table for Fuzzy Logic Final Combined Model

\begin{tabular}{|c|c|c|c|c|c|c|c|c|}
\hline \multicolumn{9}{|c|}{ Calibrated Model by Fuzzy Logic } \\
\hline \multirow{8}{*}{$\begin{array}{l}\text { Do } \\
\text { D } \\
0 \\
0 \\
0\end{array}$} & \multicolumn{7}{|l|}{ Estimated } & \multirow[t]{2}{*}{ Total observed } \\
\hline & Mode & Car & 2-wheeler & Cycle & Bus & Metro & Auto & \\
\hline & Car & 42 & 4 & 0 & 0 & 4 & 0 & 50 \\
\hline & 2-Wheeler & 10 & 101 & 0 & 2 & 3 & 0 & 116 \\
\hline & Cycle & 0 & 0 & 6 & 0 & 1 & 0 & 7 \\
\hline & Bus & 4 & 27 & 2 & 29 & 32 & 1 & 95 \\
\hline & Metro & 14 & 33 & 1 & 12 & 66 & 0 & 126 \\
\hline & Auto & 0 & 0 & 0 & 0 & 0 & $\mathbf{0}$ & 0 \\
\hline \multicolumn{2}{|c|}{ Total estimated } & 72 & 165 & 9 & 43 & 106 & 1 & 394 \\
\hline \multicolumn{4}{|c|}{ Prediction accuracy } & \multicolumn{5}{|c|}{$100 *(42+101+6+29+66) / 394=61.93 \%$} \\
\hline
\end{tabular}

Subsequently, calibrated model was validated using 212 sample size which is not used for calibration to calculate the prediction accuracy of validation samples. Validated model results are presented in Table 4.

Table 4

Validated Prediction Table for Fuzzy Logic Final Combined Model

\begin{tabular}{|c|c|c|c|c|c|c|c|c|}
\hline \multicolumn{9}{|c|}{ Validated Model by Fuzzy Logic } \\
\hline \multirow{8}{*}{$\begin{array}{l}\overrightarrow{0} \\
\stackrel{0}{0} \\
\stackrel{0}{0} \\
0 \\
0 \\
0\end{array}$} & \multicolumn{7}{|l|}{ Estimated } & \multirow{2}{*}{ Total observed } \\
\hline & Mode & Car & 2-Wheeler & Cycle & Bus & Metro & Auto & \\
\hline & Car & 25 & 2 & 0 & 0 & 1 & 0 & 28 \\
\hline & 2-Wheeler & 4 & 43 & 0 & 9 & 4 & 0 & 60 \\
\hline & Cycle & 0 & 0 & 5 & 1 & 0 & 0 & 6 \\
\hline & Bus & 1 & 17 & 2 & 22 & 17 & 1 & 60 \\
\hline & Metro & 11 & 22 & 0 & 4 & 19 & 0 & 56 \\
\hline & Auto & 0 & 0 & 0 & 0 & 2 & $\mathbf{0}$ & 2 \\
\hline \multicolumn{2}{|c|}{ Total Estimated } & 41 & 84 & 7 & 36 & 43 & 1 & 212 \\
\hline \multicolumn{3}{|c|}{ Prediction Accuracy } & \multicolumn{6}{|c|}{$100 *(25+43+5+22+19) / 212=53.77 \%$} \\
\hline
\end{tabular}

From Table 4, it can be seen that $28,60,6,60$, 56 and 2 samples are observed for selection of car, 2-wheeler, cycle, bus, metro and auto-rickshaw, respectively. But fuzzy logic mode choice model estimated total sample of $41,84,7,36,43$ and 1 with respect to 
car, 2-wheeler, cycle, bus, metro and autorickshaw, respectively. Prediction accuracy of model was estimated using number of samples correctly classified considering both observed and estimated choices. Analysis carried out demonstrates an accuracy of $53.77 \%$ of validated samples as presented in Table 4. This model also provides estimated modal split of study area for home-based work trip.

\section{Modal-Split of Study Area (Delhi) by Fuzzy Logic Technique}

Observed and estimated modal split of study area Delhi can be seen in Table 5. Above table constitutes that share of 2 -wheeler between observed \& estimated varies to an extent of $12 \%$ from $29 \%$ to $41 \%$ respectively as against share of bus decreases significantly to the tune of $13 \%$ as presented in Table 5 . Overall variation of shares of various modes of transport ranges between "-13\% to $12 \%$ " as presented in the Table 5. Following these results, further application of Fuzzy logic based mode choice model (FloMoChoMo) has been used for new transport policies to increase share of public transport service. Concerned application can be seen in succeeding section.

Table 5

Observed and Estimated Modal Split of Study Area Delhi

\begin{tabular}{|l|c|c|c|}
\hline \multirow{2}{*}{ Delhi (Combined of 3 Work Centers) } \\
\hline \multirow{2}{*}{ Modes } & \multicolumn{2}{|c|}{ Modal Split } & \multirow{2}{*}{ Variation } \\
\cline { 2 - 4 } & Observed & Estimated & \\
\hline Car & $13 \%$ & $19 \%$ & $6 \%$ \\
\hline 2-Wheeler & $29 \%$ & $41 \%$ & $12 \%$ \\
\hline Cycle & $2 \%$ & $3 \%$ & $1 \%$ \\
\hline Bus & $25 \%$ & $12 \%$ & $-13 \%$ \\
\hline Metro & $30 \%$ & $24.50 \%$ & $-6 \%$ \\
\hline Auto & $1 \%$ & $0.50 \%$ & $-1 \%$ \\
\hline Total & $\mathbf{1 0 0} \%$ & $\mathbf{1 0 0} \%$ & $\mathbf{0} \%$ \\
\hline
\end{tabular}

\section{Policy Sensitivity Analysis}

In this section, developed fuzzy logic based mode choice model (FLoMoChoMo) has been used for new transport policies. Following this, some new transport policies have been introduced in this section for improvement in share of public transport service. The estimated modal split in favor of public transport service has been observed as $36.5 \%$ in the base year. This policy sensitivity models will help to predict shift of share from personalized modes to public transport modes on basis of proposed policies in favor of public transport services.

\subsection{Evaluation of Different Policies}

Policy 1: Introducing "LRT" as new mode. Policy 2: Increasing bus frequency by $10 \%$. Policy 3: Introducing "LRT" as new mode and increasing bus frequency by $10 \%$ together.

Policy 4: Modifying bus fare structure.

Policy 5: Increasing bus frequency by $10 \%$ and modifying bus fare structure together. Policy 6: Applying Policies 1, 2 and 4 together.

\subsection{Policy Wise Improvement in Public Transport Services}

After resolving assumptions for all five polices, it is then applied to fuzzy logic based mode choice model. Therefore, it can be seen that personalized vehicle users are used to shift to public transport due to the improvement made in public transport services in the study area. Then for better improvement in public transport share, all policies have been combined together to form a new policy named as "Policy 6". The results of all policies are shown in Fig. 7. On examining the public 
transport share with respect to Policy 1 (the increase in $1.5 \%$ share is noticed). Similarly, for Policy 2, Policy 3, Policy 4 and Policy 5, the share in the public transport is gradually increased by $1.5 \%$, $3.5 \%, 3.5 \%$ and $6.5 \%$, respectively as compared to base case with respect to public transport share. All policies are then combined to form a new policy name "Policy 6". There is a significant increase in public transport share by $11.5 \%$ achieved under Policy 6. Finally, it can be seen how the public transport services with respect to before and after Policy 6 undergoes various changes in the modal share with respect to various modes of transport.

\begin{tabular}{|c|c|c|c|c|c|}
\hline Policy & Policy 1 & Policy 2 & Policy 3 & Policy 4 & Policy 5 \\
\hline $\begin{array}{c}\text { Public } \\
\text { Transport }\end{array}$ & $38.00 \%$ & $38.00 \%$ & $40.00 \%$ & $40.00 \%$ & $43.00 \%$ \\
\hline \multirow[t]{4}{*}{$\begin{array}{c}\text { Variation } \\
(\%)\end{array}$} & $1.5 \%$ & $1.5 \%$ & $3.5 \%$ & $3.5 \%$ & $6.5 \%$ \\
\hline & & \multicolumn{2}{|c|}{ Policy 6} & & \\
\hline & & $\begin{array}{c}\text { Public } \\
\text { Transport }\end{array}$ & $48.00 \%$ & $\downarrow$ & \\
\hline & & $\begin{array}{c}\text { Variation } \\
(\%)\end{array}$ & $11.5 \%$ & & \\
\hline
\end{tabular}

Fig. 7.

Policy-Wise Improvement in Public Transport System

\subsection{Impact on Private Vehicle Modal Split in Policy 6}

Table 6 presents a comparative analysis of change in shift of share for all modes of transport with respect to before and after Policy 6. Table 6 states that by introducing LRT as new mode of public transport, other modes shares including personalized vehicle (two-wheeler and car) shift to LRT, i.e. public transport. The variation in modal split of all the modes including LRT shows that commuters have willingness to shift to other modes of transport based on trip characteristics. For example, in Policy 6 travel behavior of commuter have been influenced by "Travel Cost" and "Out-of Vehicle Travel Time", which shows that by introducing other efficient policies the modal share of public transport may be improved. 
Table 6

Modal Split of Study Area Delhi before and after Application of Policy 6

\begin{tabular}{|l|c|c|c|c|}
\hline \multirow{2}{*}{ Mode } & \multicolumn{2}{|c|}{ Modal Split } & \multirow{2}{*}{ Shift in Share to LRT } & Change in Modal Split \\
\cline { 2 - 5 } & Before Policy 6 & After Policy 6 & $-2.00 \%$ & $3.00 \%$ \\
\hline 2-Wheeler & $19 \%$ & $16 \%$ & $-6.00 \%$ & $6.00 \%$ \\
\hline Cycle & $41 \%$ & $35 \%$ & $-2.00 \%$ & $2.00 \%$ \\
\hline LRT & - & $1 \%$ & - & - \\
\hline Bus & $12 \%$ & $10 \%$ & $4.00 \%$ & $2.00 \%$ \\
\hline Metro & $24.50 \%$ & $20 \%$ & $6.00 \%$ & $4.50 \%$ \\
\hline Auto & $0.50 \%$ & $0 \%$ & $0.00 \%$ & $0.50 \%$ \\
\hline Total & $\mathbf{1 0 0} \%$ & $\mathbf{1 0 0} \%$ & $\mathbf{0 . 0 0} \%$ & $\mathbf{1 8 . 0 0} \%$ \\
\hline
\end{tabular}

Note: The symbol '-' shown in Table 6 states that LRT is not present in base year scenario.

\section{Conclusions}

In this case study, fuzzy logic technique has been used to develop mode choice model on basis of trip characteristics of commuters by using a numbers of variables affecting the mode choice behavior of commuters with respect to different alternative modes in specific choice set. Finally the study is conclusive with the following:

1. There is a shift in the modal share to the extent of $14 \%$ in favor of introduction of LRT under Policy 1.

2. The share of bus commuters increases to extent of $5 \%$ with the increase in the frequency of bus by $10 \%$ under Policy 2 .

3. The combined impact of new mode LRT and increase in the frequency of bus service by $10 \%$ results in the overall increase in share of public transport service by $3.5 \%$ under Policy 3 .

4. This is worth mentioning that the lower bus fare for bus operations results in the increase in the share of bus commuters by $6 \%$. This is primarily due to the $3.5 \%$ shift of the personalized vehicle users to public transport service under Policy 4.
5. The combined effect of lower bus fare along with increase in the bus frequency by $10 \%$ results in the increase of public transport share by $6.5 \%$ under Policy 5. This is primarily due to the shift of $5.5 \%$ share from personalized modes to public transport service.

6. Policy 6 includes all the types of improvement for public transport system and is found to be the best as compared to all the individual policies, resulting with the increase of $11.5 \%$ of public transport share as against the present situation. This implies that public transport share is increased from $36.5 \%$ (before Policy 6) to $48 \%$ (after Policy 6).

7. The fuzzy logic based mode choice model (FLoMoChoMo) offers a significant flexibility in evaluating any kind of sensitive policy with regard to public transport system. Though, this study was confined to testing six policies, if more policies with regard to the promotion of public transport system is envisaged, this model has a capability to estimate confidently the increase in modal with respect to public transport system. 
8. The development of fuzzy logic based mode choice model using trial and error method is a time consuming process. It is concluded that fuzzy logic technique gives satisfactory results by using trial and error method. The completion of development of FloMoChoMo model with 3000 fuzzy if-then rules took almost 250 man hours.

\section{Appendix 1 - Glossary of Terms}

\begin{tabular}{|c|c|}
\hline $\mathbf{A}$ & Income Coefficient \\
\hline$a_{k}$ & "k" number of coefficient \\
\hline ANN & Artificial Neural Network \\
\hline AVG & Average \\
\hline B & OVTT Coefficient \\
\hline BG & Big \\
\hline BRTS & Bus Rapid Transit System \\
\hline $\mathbf{C}$ & IVTT Coefficient \\
\hline CFT & Comfort \\
\hline $\mathbf{C P}$ & Connaught Place \\
\hline CRRI & Central Road Research Institute \\
\hline $\mathbf{D}$ & Travel Cost Coefficient \\
\hline DCM & Discrete Choice Model \\
\hline DTC & Delhi Transport Corporation \\
\hline DMRC & Delhi Metro Rail Corporation \\
\hline $\mathbf{E}$ & Comfort Level Coefficient \\
\hline FIS & Fuzzy Inference System \\
\hline FL & Fuzzy Logic \\
\hline FLS & Fuzzy Logic System \\
\hline FLoMoChoMo & Fuzzy Logic based Mode Choice Model \\
\hline GR & Gravity \\
\hline HBW & Home Based Work Trip \\
\hline HG & High \\
\hline INC & Income \\
\hline IVTT & In-Vehicle Travel Time \\
\hline LC & Latent Class \\
\hline LG & Large \\
\hline LRT & Light Rail Transit \\
\hline LS & Less \\
\hline LW & Low \\
\hline MED & Medium \\
\hline Min & Minimum \\
\hline $\min$ & Minutes \\
\hline MNL & Multi-Nomial Logit \\
\hline MRTS & Mass Rapid Transit System \\
\hline MXL & Mixed Logit \\
\hline NTS & New Transit System \\
\hline NSP & Netaji Subhash Place \\
\hline
\end{tabular}




\begin{tabular}{|c|c|}
\hline NP & Nehru place district center \\
\hline OVTT & Out-of-Vehicle Travel Time \\
\hline $\mathbf{P}$ & Probability \\
\hline PT & Public Transport \\
\hline $\mathbf{P}_{\text {in }}$ & Probability of individual " $i$ " selecting mode " $n$ " \\
\hline $\mathbf{R}_{i}$ & $\mathrm{i}^{\text {th }}$ Fuzzy IF-THEN rule \\
\hline $\mathbf{R P}$ & Revealed Preference \\
\hline SL & Small \\
\hline SP & Stated Preference \\
\hline TC & Travel Cost \\
\hline TCM & Transportation Congestion Management \\
\hline TLFD & Trip Length Frequency Distribution \\
\hline TW & Two Wheeler \\
\hline $\mathbf{U}$ & Utility Of Mode \\
\hline $\mathbf{U}_{\text {in }}$ & Utility function of mode " $n$ " for individual “" \\
\hline $\mathbf{U}_{\mathrm{mi}}$ & Net Utility of mode " $m$ " for individual " $i$ " \\
\hline UTL & Utility \\
\hline VBG & Very Big \\
\hline VHG & Very High \\
\hline VLG & Very Large \\
\hline VLS & Very Less \\
\hline VLW & Very Low \\
\hline VSL & Very Small \\
\hline w.r.t & With Respect To \\
\hline $\mathbf{X}_{\mathrm{i}}$ & $\mathrm{i}^{\text {th }}$ input variable \\
\hline $\mathbf{y}$ & Output \\
\hline$\mu$ & Membership value \\
\hline$\mu_{\mathrm{A}}$ & Membership function of Fuzzy set "A" \\
\hline$\eta$ & Location Parameter \\
\hline$\chi_{\text {mik }}$ & " $k$ " number of attributes of mode " $m$ " for individual " $i$ " \\
\hline$\cap$ & Intersection operator \\
\hline
\end{tabular}




\section{References}

Akiyama, T.; Okushima, M. 2010. Fuzzy travel behaviour model with spatial information to evaluate public transport policy. In Proceedings of the the 12th World Conference on Transportation Research, Portugal.

Dhingra, S.L.; Ramanajee, M. 1998. Evaluation of Utility of Mode Using Fuzzy Integrals. In Proceeding of the 8th World Conference on Transportation Research, Belgium.

Hellmann, M. 2000. Fuzzy Logic Introduction. Universit'e de Rennes 1, UFR S.P.M, Campus de Beaulieu.

Madhu, D. 2008. Fuzzy Logic Based Micro-Scopic Traffic Simulation Model for Transport Policy Evaluation. Gifu University, Japan: PhD Thesis.

Nath, B. 2008. Travel mode choice behaviour modeling by a non-conventional approach using fuzzy measures, IIT Delhi, India: $\mathrm{PhD}$ Thesis.

Postorino, M.N.; Versaci, M. 2008. A fuzzy approach to simulate the user mode choice behaviour. Italy: Mediterranean University of Reggio Calabria. 